

\begin{tabular}{|c|l|}
\hline Title & $\begin{array}{l}\text { Spontaneous Lorentz symmetry breaking by an } \\
\text { antisymmetric tensor field }\end{array}$ \\
\hline Author(s) & Yokoi, Naoto; Higashijima, Kiyoshi \\
\hline Citation & Physical Review D. 64(2) p. 025004 \\
\hline Issue Date & $2001-06$ \\
\hline oaire:version & VoR \\
\hline URL & https://hdl. handle. net/11094/3034 \\
\hline rights & $\begin{array}{l}\text { Higashijima, Kiyoshi, Yokoi, Naoto, Physical } \\
\text { Review } D, 64,2,025004,2001-06 . \text { "Copyright } \\
2001 \text { by the American Physical Society." }\end{array}$ \\
\hline Note & \multicolumn{2}{|l}{} \\
\hline
\end{tabular}

Osaka University Knowledge Archive : OUKA

https://ir. Library. osaka-u. ac. jp/

Osaka University 


\title{
Spontaneous Lorentz symmetry breaking by an antisymmetric tensor field
}

\author{
Kiyoshi Higashijima* and Naoto Yokoi ${ }^{\dagger}$ \\ Department of Physics, Graduate School of Science, Osaka University, Toyonaka, Osaka 560-0043, Japan
}

(Received 7 February 2001; published 6 June 2001)

\begin{abstract}
We study spontaneous Lorentz symmetry breaking in a field theoretical model in $2+1$ dimensions, inspired by string theory. This model is a gauge theory of an antisymmetric tensor field and a vector field (photon). The Nambu-Goldstone (NG) boson for the spontaneous Lorentz symmetry breaking is identified with the unphysical massless photon in covariant quantization. We also discuss an analog of the equivalence theorem between the amplitudes for emission or absorption of the physical massive antisymmetric tensor field and those of the unphysical massless photon. The low-energy effective action of the NG boson is also discussed.
\end{abstract}

DOI: 10.1103/PhysRevD.64.025004

PACS number(s): 11.30.Cp, 11.10.Kk, 11.30.Qc

\section{INTRODUCTION}

Quantum field theories based on Poincaré invariance, in particular, Lorentz invariance successfully describe elementary particles below the weak scale energy $(\sim 100 \mathrm{GeV})$. In the last few years, a possible type of Lorentz noninvariant extension of quantum field theories has been extensively studied. These are the field theories on a spacetime whose coordinates are noncommutative, called noncommutative field theories [1-4]. The action of the noncommutative field theories can be constructed by replacing the product of fields in the action of the ordinary field theory with the $\star$ product defined as

$$
\left.f(x) \star g(x) \equiv \exp \left[i \theta^{i j} \frac{\partial}{\partial \xi^{i}} \frac{\partial}{\partial \eta^{j}}\right] f(x+\xi) g(x+\eta)\right|_{\xi=\eta=0}
$$

where $\theta^{i j}$ is a constant noncommutative parameter: $\left[x^{i}, x^{j}\right]$ $=i \theta^{i j}$. Thus the action explicitly contains the constant antisymmetric tensor $\theta^{i j}$, and the Lorentz invariance in $p+1$ dimensions for $p \geqslant 2$ cannot be maintained.

String theory naturally provides the noncommutative field theories as the world volume effective theories on D branes [4]; the world volume effective theory of $\mathrm{D} p$-brane with a constant background Neveu-Schwarz-Neveu-Schwarz (NSNS) $B$ field is equivalent to a $(p+1)$-dimensional noncommutative field theory whose constant noncommutative parameter $\theta^{i j}$ is given by the background NS-NS $B$ field $B_{i j}$. In string theory the NS-NS $B$ field is indeed a dynamical field in a closed string sector and thus the constant background field can be interpreted as the constant vacuum expectation value of the dynamical NS-NS $B$ field. From this perspective, the Lorentz symmetry is spontaneously broken by the constant vacuum expectation value of the second rank antisymmetric tensor field.

In this paper, based on this viewpoint, we discuss the spontaneous Lorentz symmetry breaking within the effective-field theory of the string theory. Concretely, we in-

\footnotetext{
*Email address: higashij@ @et.phys.sci.osaka-u.ac.jp

${ }^{\dagger}$ Email address: yokoi@het.phys.sci.osaka-u.ac.jp
}

vestigate the Nambu-Goldstone $(\mathrm{NG})$ boson for the Lorentz symmetry breaking in a field theoretical toy model in $2+1$ dimensions of a second rank antisymmetric tensor field and a vector field, which is inspired by the effective theory of the string theory. ${ }^{1}$ We find that the NG boson is an unphysical field and their amplitudes, however, provide useful information about the physical amplitudes of the model through the "'equivalence theorem.', We also discuss the low-energy dynamics of the NG boson from the perspective of the nonlinear realization of the Lorentz symmetry.

This paper is organized as follows. In Sec. II, we introduce the gauge invariant model of a second rank antisymmetric tensor field and a vector field and discuss the covariant canonical quantization of the model. In Sec. III, the vacuum of the model where the antisymmetric tensor field has a vacuum expectation value is discussed, and also the NG boson for the spontaneous Lorentz symmetry breaking is studied in detail. In Sec. IV, a possible perturbation of the model is discussed and the equivalence theorem between the physical amplitudes and the amplitudes of the unphysical NG boson is also argued. In Sec. V, some related problems are discussed and the relation to the noncommutative field theories is speculated.

\section{A TOY MODEL FOR FIELD THEORY OF $B_{\mu \nu}$ AND $A_{\mu}$}

In this section we discuss the covariant canonical quantization of a toy model for the gauge-invariant field theory of a second rank antisymmetric tensor field $B_{\mu \nu}$ coupled with a vector field $A_{\mu}$ (photon) in $2+1$ dimension.

\section{A. Canonical quantization of the model}

The action of the toy model is given $b y^{2}$

$$
S=\int d^{3} x\left[\frac{1}{12 m^{2}}\left(H_{\mu \nu \rho}\right)^{2}-\frac{1}{4}\left(F_{\mu \nu}-B_{\mu \nu}\right)^{2}\right],
$$

\footnotetext{
${ }^{1}$ Spontaneous breaking of the Lorentz symmetry has been discussed previously in gravity theory with the torsion by introducing quadratic terms of the curvature and torsion [5]. In this paper, we confine ourselves to the Lorentz symmetry breaking in a pure gauge theory neglecting the effect of the gravitation.

${ }^{2}$ The metric is $\eta_{\mu \nu}=\eta^{\mu \nu}=\operatorname{diag}(+1,-1,-1)$.
} 
where

$$
H_{\mu \nu \rho}=\partial_{\mu} B_{\nu \rho}+\partial_{\rho} B_{\mu \nu}+\partial_{\nu} B_{\rho \mu}, \quad F_{\mu \nu}=\partial_{\mu} A_{\nu}-\partial_{\nu} A_{\mu},
$$

and $m$ is a parameter with dimension of mass. This action is inspired by string theory. ${ }^{3}$ Indeed, the first and second terms in Eq. (2.1) are the same form as the leading term of the effective action of $B_{\mu \nu}$, which is a massless mode of closed string, and the leading term of the Dirac-Born-Infeld (DBI) action of D-brane world volume effective theory, which is the effective action of the open string sector in $\alpha^{\prime}$ expansion [10].

The action (2.1) is invariant under the gauge transformation

$$
\begin{gathered}
\delta B_{\mu \nu}(x)=\partial_{\mu} \Lambda_{\nu}(x)-\partial_{\nu} \Lambda_{\mu}(x), \\
\delta A_{\mu}(x)=\Lambda_{\mu}(x)+\partial_{\mu} \Lambda(x),
\end{gathered}
$$

where $\Lambda_{\mu}(x)$ and $\Lambda(x)$ are one-form and scalar gauge functions, respectively. Because of this gauge invariance, the system described by the action (2.1) is a singular (constrained) system. Thus, for the canonical quantization, one must introduce gauge fixing terms. Since we want to discuss the spontaneous Lorentz symmetry breaking in the sequel, we must take Lorentz gauge-invariant fixing terms. We introduce the following gauge fixing terms:

$$
S_{\mathrm{gf}}=\int d^{3} x\left(C^{\nu} \partial^{\mu} B_{\mu \nu}-B \partial_{\mu} A^{\mu}-C \partial_{\mu} C^{\mu}\right),
$$

where $B(x)$ is the Nakanishi-Lautrup (NL) $B$ field for the vector field and $C_{\mu}(x)$ and $C(x)$ are the counterparts for the antisymmetric tensor gauge field $[11,12]$. These gauge fixing terms are the analogs of the Landau gauge in quantum electrodynamics (QED).

Although the canonical quantization of the model in the Becchi-Rouet-Stora-Tyutin (BRST) formalism can be carried out, we make the canonical quantization in the NL formalism $[11,12]$ for simplicity. ${ }^{4}$

The gauge fixed action is given by Eqs. (2.1) and (2.4):

$$
\begin{aligned}
S_{\text {total }}= & \int d^{3} x \mathcal{L}_{\text {total }}(x) \\
= & \int d^{3} x\left[\frac{1}{12 m^{2}}\left(H_{\mu \nu \rho}\right)^{2}-\frac{1}{4}\left(F_{\mu \nu}-B_{\mu \nu}\right)^{2}\right. \\
& \left.+C^{\nu} \partial^{\mu} B_{\mu \nu}-B \partial_{\mu} A^{\mu}-C \partial_{\mu} C^{\mu}\right] .
\end{aligned}
$$

${ }^{3}$ In fact, this type of action appears in various contexts of string theory [6-9].

${ }^{4}$ In the BRST formalism, in addition, ghost and antighost fields are introduced. However, since this action is a quadratic action with Abelian gauge symmetry, ghost and antighost fields are free fields and decouple.
The equations of motion derived from Eq. (2.5) for each field become as follows:

$$
\begin{aligned}
B_{\mu \nu}: & \quad \frac{1}{m^{2}} \partial_{\rho} H^{\rho \mu \nu}+\left(B^{\mu \nu}-F^{\mu \nu}+C^{\mu \nu}\right)=0, \\
A_{\mu}: & -\partial_{\rho}\left(F^{\rho \mu}-B^{\rho \mu}\right)-\partial^{\mu} B=0 \\
C_{\mu}: & -\partial_{\rho} B^{\rho \mu}-\partial^{\mu} C=0 \\
B: & \partial_{\mu} A^{\mu}=0 \\
C: & \partial_{\mu} C^{\mu}=0
\end{aligned}
$$

where $C_{\mu \nu}=\partial_{\mu} C_{\nu}-\partial_{\nu} C_{\mu}$. Actually, by combining these equations, one can find free field equations of each field:

$$
\begin{aligned}
& \square^{2} A_{\mu}=0, \quad \square\left(\square+m^{2}\right) B_{\mu \nu}=0, \\
& \square^{2} C_{\mu}=0, \quad \square B=0, \quad \square C=0 .
\end{aligned}
$$

Thus this model is essentially a free field theory and can be quantized completely. Note that the antisymmetric tensor field $B_{\mu \nu}$ is a mixture of massive and massless components. Following the procedure in Refs. [11,12], the threedimensional commutation relations can be calculated by using the equal-time commutation relations

$$
\begin{gathered}
{\left[\phi_{I}(\boldsymbol{x}, t), \phi_{J}(\boldsymbol{y}, t)\right]=0, \quad\left[\pi^{I}(\boldsymbol{x}, t), \pi^{J}(\boldsymbol{y}, t)\right]=0,} \\
{\left[\phi_{I}(\boldsymbol{x}, t), \pi^{J}(\boldsymbol{y}, t)\right]=i \delta_{I}^{J} \delta^{2}(\boldsymbol{x}-\boldsymbol{y}),} \\
\text { where } \quad \pi^{I}(\boldsymbol{x}, t) \equiv \frac{\partial \mathcal{L}_{\text {total }}(x)}{\partial \dot{\boldsymbol{\phi}}_{I}(\boldsymbol{x}, t)} .
\end{gathered}
$$

Here we abbreviate various fields as $\phi_{I}(\boldsymbol{x}, t)$, where $I$ denotes various indices. The explicit forms of the nonvanishing three-dimensional commutation relations are ${ }^{5}$

$$
\begin{aligned}
{\left[B(x), A_{\mu}(y)\right]=} & {\left[C(x), C_{\mu}(y)\right]=-i \partial_{\mu}^{x} D(x-y), } \\
{\left[A_{\mu}(x), A_{\nu}(y)\right]=} & {\left[C_{\mu}(x), A_{\nu}(y)\right] } \\
= & -i \eta_{\mu \nu} D(x-y)+i \partial_{\mu}^{x} \partial_{\nu}^{x} E(x-y), \\
{\left[C_{\mu}(x), B_{\nu \rho}(y)\right]=} & -i\left(\eta_{\mu \nu} \partial_{\rho}^{x}-\eta_{\mu \rho} \partial_{\nu}^{x}\right) D(x-y), \\
{\left[B_{\mu \nu}(x), B_{\rho \sigma}(y)\right]=} & i\left[\eta_{\mu \rho} \partial_{\nu}^{x} \partial_{\sigma}^{x}-\eta_{\mu \sigma} \partial_{\nu}^{x} \partial_{\rho}^{x}-\eta_{\nu \rho} \partial_{\mu}^{x} \partial_{\sigma}^{x}\right. \\
& \left.+\eta_{\nu \sigma} \partial_{\mu}^{x} \partial_{\rho}^{x}+m^{2}\left(\eta_{\mu \rho} \eta_{\nu \sigma}-\eta_{\mu \sigma} \eta_{\nu \rho}\right)\right] \\
& \times \Delta\left(x-y: m^{2}\right)-i\left(\eta_{\mu \rho} \partial_{\nu}^{x} \partial_{\sigma}^{x}\right. \\
& -\eta_{\mu \sigma} \partial_{\nu}^{x} \partial_{\rho}^{x}-\eta_{\nu \rho} \partial_{\mu}^{x} \partial_{\sigma}^{x} \\
& \left.+\eta_{\nu \sigma} \partial_{\mu}^{x} \partial_{\rho}^{x}\right) D(x-y),
\end{aligned}
$$

\footnotetext{
${ }^{5}$ The equal-time commutation relations are obtained by setting $x^{0}=y^{0}$ in the three-dimensional commutation relations.
} 
where

$$
\begin{aligned}
\Delta\left(x: m^{2}\right) & \equiv \frac{1}{(2 \pi)^{2} i} \int d^{3} k \quad \epsilon\left(k_{0}\right) \delta\left(k^{2}-m^{2}\right) e^{-i k x}, \\
D(x) & \equiv \Delta\left(x: m^{2}=0\right), \\
E(x) & \equiv \frac{1}{(2 \pi)^{2} i} \int d^{3} k \quad \epsilon\left(k_{0}\right) \delta^{\prime}\left(k^{2}\right) e^{-i k x}, \\
\square E(x) & =D(x) .
\end{aligned}
$$

In order to quantize the model consistently, we require the physical state conditions analogous to the ordinary QED in the NL formalism $[11,12]$. We define the physical state through the physical state conditions:

$$
\begin{aligned}
& \left.\left.C_{\mu}^{(+)}(x) \mid \text { phys }\right\rangle=0, \quad B^{(+)}(x) \mid \text { phys }\right\rangle=0, \\
& \left.C^{(+)}(x) \mid \text { phys }\right\rangle=0,
\end{aligned}
$$

where $\phi_{I}^{(+)}(x)$ means the positive energy part of $\phi_{I}(x)$. In the gauge (2.4), as seen from Eq. (2.11), $C_{\mu}(x)$ is a dipole field. Although the separation between the positive and negative energy part of a dipole field is a nontrivial problem, the cutoff procedure is known to give the well-defined separation as found in Sec. II B $[12,13]$. Thus the physical state conditions (2.16) are well defined.

\section{B. The physical spectrum}

In order to find the spectrum of the model, we define the creation and annihilation operators of each field. The annihilation operators are defined by the Fourier transforms:

$$
\begin{gathered}
C^{(+)}(x)=\frac{1}{2 \pi} \int d^{3} k \theta\left(k_{0}\right) e^{-i k x} b(k), \\
B^{(+)}(x)=\frac{1}{2 \pi} \int d^{3} k \theta\left(k_{0}\right) e^{-i k x} c(k), \\
C_{\mu}^{(+)}(x: \epsilon)=\frac{1}{2 \pi} \int d^{3} k \theta\left(k_{0}-\epsilon\right) e^{-i k x} c_{\mu}(k), \\
A_{\mu}^{(+)}(x: \epsilon)=\frac{1}{2 \pi} \int d^{3} k \theta\left(k_{0}-\epsilon\right) e^{-i k x} a_{\mu}(k), \\
B_{\mu \nu}^{(+)}(x)=\frac{1}{2 \pi} \int d^{3} k \theta\left(k_{0}\right) e^{-i k x} b_{\mu \nu}(k),
\end{gathered}
$$

and the creation operators are defined by the Hermitian conjugate of Eq. (2.17). $\epsilon$ in the definition (2.17) is an infrared cutoff parameter for the dipole fields $[12,13]$.

The commutation relations of the operators can be calculated by the three-dimensional commutation relations (2.13). The nonvanishing commutation relations are

$$
\begin{aligned}
{\left[b(p), a_{\mu}^{+}(k)\right]=} & {\left[c(p), c_{\mu}^{+}(k)\right] } \\
= & i p_{\mu} \theta\left(p_{0}\right) \delta\left(p^{2}\right) \delta^{3}(p-k), \\
{\left[a_{\mu}(p), a_{\nu}^{+}(k)\right]=} & {\left[c_{\mu}(p), a_{\nu}^{+}(k)\right] } \\
= & -\eta_{\mu \nu} \theta\left(p_{0}\right) \delta\left(p^{2}\right) \delta^{3}(p-k) \\
& -p_{\mu} p_{\nu} \theta\left(p_{0}\right) \delta^{\prime}\left(p^{2}\right) \delta^{3}(p-k), \\
{\left[c_{\mu}(p), b_{\nu \rho}^{+}(k)\right]=} & i\left(\eta_{\mu \nu} p_{\rho}-\eta_{\mu \rho} p_{\nu}\right) \theta\left(p_{0}\right) \delta\left(p^{2}\right) \delta^{3}(p-k), \\
{\left[b_{\mu \nu}(p), b_{\rho \sigma}^{+}(k)\right]=} & {\left[-\eta_{\mu \rho} p_{\nu} p_{\sigma}+\eta_{\mu \sigma} p_{\nu} p_{\rho}+\eta_{\nu \rho} p_{\mu} p_{\sigma}\right.} \\
& -\eta_{\nu \sigma} p_{\mu} p_{\rho}+m^{2}\left(\eta_{\mu \rho} \eta_{\nu \sigma}\right. \\
& \left.\left.-\eta_{\mu \sigma} \eta_{\nu \rho}\right)\right] \theta\left(p_{0}\right) \delta\left(p^{2}-m^{2}\right) \delta^{3}(p-k) \\
& -\left(-\eta_{\mu \rho} p_{\nu} p_{\sigma}+\eta_{\mu \sigma} p_{\nu} p_{\rho}+\eta_{\nu \rho} p_{\mu} p_{\sigma}\right. \\
& \left.-\eta_{\nu \sigma} p_{\mu} p_{\rho}\right) \theta\left(p_{0}\right) \delta\left(p^{2}\right) \delta^{3}(p-k) .
\end{aligned}
$$

In terms of these operators, the physical state conditions (2.16) become

$$
\left.\left.\left.c_{\mu}(p) \mid \text { phys }\right\rangle=0, \quad b(p) \mid \text { phys }\right\rangle=0, \quad c(p) \mid \text { phys }\right\rangle=0 .
$$

The vacuum state is defined by

$$
\begin{aligned}
b_{\mu \nu}(p)|\mathrm{vac}\rangle & =0, \quad a_{\mu}(p)|\mathrm{vac}\rangle=0, \\
c_{\mu}(p)|\mathrm{vac}\rangle & =0, \quad b(p)|\mathrm{vac}\rangle=0, \\
c(p)|\mathrm{vac}\rangle & =0 .
\end{aligned}
$$

The vacuum state is physical by definition. One-particle states are constructed by the creation operators from the vacuum state. Physical one-particle states, which satisfy condition (2.19), are constructed by the creation operators, which commute with $c_{\mu}(p), b(p)$, and $c(p)$. The physical states are summarized as follows: ${ }^{6}$

(i) Physical massless states in the momentum frame $p_{\mu}$ $=(p, 0, p)$,

$$
c_{1}^{+}(p)|\mathrm{vac}\rangle, \quad\left[c_{0}^{+}(p)-c_{2}^{+}(p)\right]|\mathrm{vac}\rangle, \quad b^{+}(p)|\mathrm{vac}\rangle .
$$

(ii) Physical massive states with mass $m$ in the rest frame $p_{\mu}=(m, 0,0)$,

$$
u_{\mu \nu}^{+}(p)|\mathrm{vac}\rangle \equiv\left[b_{\mu \nu}^{+}(p)-f_{\mu \nu}^{+}(p)+c_{\mu \nu}^{+}(p)\right]|\mathrm{vac}\rangle .
$$

Here $u_{\mu \nu}^{+}(p)$ is the creation operator of the gauge-invariant field $U_{\mu \nu}(x) \equiv B_{\mu \nu}(x)-F_{\mu \nu}(x)+C_{\mu \nu}(x)$. Note that the

\footnotetext{
${ }^{6} f_{\mu \nu}(p)$ and $c_{\mu \nu}(p)$ are the Fourier transforms of $F_{\mu \nu}^{(+)}(x: \epsilon)$ and $C_{\mu \nu}^{(+)}(x: \epsilon)$, respectively.
} 
massless states of photon $a_{\mu}(p)$ are all unphysical due to the "large" gauge symmetry with one-form gauge function (2.3).

One can show that all the physical massless states (2.22) are null states from the commutation relations (2.18). The physical massive state $u_{12}^{+}(p)|\mathrm{vac}\rangle$ is the propagating state with positive norm and $u_{01}^{+}(p)|\mathrm{vac}\rangle$ and $u_{02}^{+}(p)|\mathrm{vac}\rangle$ are null states in the rest frame. Thus we conclude that the physical propagating degree of freedom ${ }^{7}$ of the model is a physical massive state $u_{12}^{+}(p) \mid$ vac $\rangle$ with mass $m$. Although the action (2.1) has the gauge symmetry (2.3), the physical massive state appears through the generalized Stueckelberg formalism, which is the antisymmetric tensor field version of the Stueckelberg formalism of QED [14]. The antisymmetric tensor field $B_{\mu \nu}$ " "eats" the degrees of freedom of the gauge field $A_{\mu}$ and become a massive antisymmetric tensor field. Note that a massless second rank antisymmetric tensor field has no physical propagating degrees of freedom and a massive one has one physical propagating degree in $2+1$ dimension. ${ }^{8}$

We consider the interesting limit of the model, $m \rightarrow 0$. This corresponds to the limit where the modes of closed string decouple in the corresponding effective action of string theory discussed in Sec. II A. In this limit, the commutation relation of $B_{\mu \nu}$ in Eq. (2.13) becomes

$$
\left[B_{\mu \nu}(x), B_{\rho \sigma}(y)\right]=0,
$$

and the commutators of the creation and annihilation operators also become

$$
\left[b_{\mu \nu}(p), b_{\rho \sigma}^{+}(k)\right]=0 \text {. }
$$

Thus the states associated with the antisymmetric tensor field $B_{\mu \nu}$ become zero norm. In this limit, the physical propagating massless state in the momentum frame $p_{\mu}=(p, 0, p)$ is given by

$$
\left[u_{01}^{+}(p)-u_{12}^{+}(p)\right]|\mathrm{vac}\rangle \text {. }
$$

Indeed, the norm of this physical propagating state becomes

$$
\begin{aligned}
& \left\langle\left[u_{01}(p)-u_{12}(p)\right]\left[u_{01}^{+}(p)-u_{12}^{+}(p)\right]\right\rangle \\
& \quad=\left\langle\left[f_{01}(p)-f_{12}(p)\right]\left[f_{01}^{+}(p)-f_{12}^{+}(p)\right]\right\rangle \\
& \quad=4 p^{2}\left\langle a_{1}(p) a_{1}^{+}(p)\right\rangle .
\end{aligned}
$$

Hence, in this limit, the physical propagating state becomes essentially the transverse photon $a_{1}^{+}(p) \mid$ vac $\rangle$. However it is worth noting that even though the norms of $\left[u_{01}^{+}(p)\right.$ $\left.-u_{12}^{+}(p)\right]|\mathrm{vac}\rangle$ and $\left[f_{01}^{+}(p)-f_{12}^{+}(p)\right]|\mathrm{vac}\rangle$ are the same in

\footnotetext{
${ }^{7}$ In this paper, the physical propagating state means the physical state with positive norm, which contributes to the physical amplitudes.

${ }^{8} \mathrm{~A}$ massless photon also has one physical propagating degree in $(2+1)$ dimensions.
}

the limit $m \rightarrow 0$, the physical propagating state is not $\left[f_{01}^{+}(p)-f_{12}^{+}(p)\right]|\mathrm{vac}\rangle$, but $\left[u_{01}^{+}(p)-u_{12}^{+}(p)\right]|\mathrm{vac}\rangle$.

This situation is similar to the broken phase of the YangMills-Higgs model, where the equivalence theorem holds $[15,16]$. This theorem claims the amplitude for emission or absorption of the longitudinal states of the massive gauge boson becomes equal, at high energy, to the amplitude for emission or absorption of the unphysical Nambu-Goldstone states, which is absorbed by the gauge boson. In our model, the physical massive state of the antisymmetric tensor field appears after the antisymmetric tensor field "eats" the unphysical state of the transverse photon. The above analysis of the norm of the physical states implies that the analogous equivalence theorem holds in our model; in the high-energy region where one can ignore mass $m$, the amplitude for emission or absorption of the longitudinal states of the physical massive antisymmetric tensor field is the same as the amplitude for emission or absorption of the unphysical massless transverse photon.

\section{SPONTANEOUS LORENTZ SYMMETRY BREAKING BY THE ANTISYMMETRIC TENSOR FIELD}

In this section we discuss the spontaneous breaking of the Lorentz symmetry by a constant vacuum expectation value (VEV) of the second rank antisymmetric tensor field in our model.

\section{A. The Nambu-Goldstone boson for the spontaneous Lorentz symmetry breaking}

The equations of motion (2.6)-(2.10) have a solution such that only $B_{\mu \nu}$ and $F_{\mu \nu}$ have constant nonzero VEV's: ${ }^{9}$

$$
\left\langle B_{12}\right\rangle=\left\langle F_{12}\right\rangle=B_{\mathrm{VEV}}=\text { const }(\neq 0) .
$$

One can easily find that this solution is a ground state with vanishing energy of the Hamiltonian derived from the action (2.5). [See Eq. (3.3).] Although $B_{\mathrm{VEV}}$ is an undetermined constant in our model, we assume this to be a nonzero constant in the sequel. Since the VEV's of $C_{\mu}, B$, and $C$ vanish, this vacuum is a physical state. In the framework of the nonperturbative string theory, the possibility of $\left\langle B_{\mu \nu}\right\rangle \neq 0$ has been discussed in Refs. $[17,18]$. In this viewpoint, the nonzero $\mathrm{VEV}$ of $B_{12}$ induces a spontaneous magnetization $\left\langle F_{12}\right\rangle$.

Since $B_{\mu \nu}$ and $F_{\mu \nu}$ are not gauge invariant under the gauge transformation (2.3), one may expect to be able to eliminate this VEV by the gauge transformation. However the gauge transformation that eliminates the $\mathrm{VEV}$ requires the linear one-form gauge functions such as $\Lambda_{1}(x)$ $=\frac{1}{2} B_{\mathrm{VEV}} x^{2}$ and $\Lambda_{2}(x)=-\frac{1}{2} B_{\mathrm{VEV}} x^{1}$. These gauge functions are ill-defined at infinity. Hence we do not require the invari-

\footnotetext{
${ }^{9}$ If the $\operatorname{VEV}\left\langle\widetilde{B}^{\mu}\right\rangle\left(\equiv \frac{1}{2} \epsilon^{\mu \nu \rho}\left\langle B_{\nu \rho}\right\rangle\right)=\left\langle\widetilde{F}^{\mu}\right\rangle$ is a time-like constant vector, one can transform it to this form by an appropriate Lorentz transformation.
} 
ance under such singular gauge transformations to define the Hilbert space of the quantum theory.

As discussed in Ref. [19], on the vacuum (3.1), the $(2+1)$-dimensional Lorentz symmetry $S O(2,1) \sim S L(2, \mathbf{R})$ is spontaneously broken down to the spatial rotation $S O(2) \sim U(1)$ by the VEV of $B_{12}$ and $F_{12} \cdot{ }^{10}$ What are the NG bosons for the broken boost generators in this model?

In order to answer this question, we construct the generators of the Lorentz transformations $M_{\rho \sigma}$ by the Noether method. The conserved currents $M_{\rho \sigma}{ }^{\mu}(x)$ for the Lorentz symmetry can be derived from the action (2.5): ${ }^{11}$

$$
\begin{aligned}
M_{\rho \sigma}^{\mu}(x)= & x_{\rho} T_{\sigma}^{\mu}(x)-x_{\sigma} T_{\rho}^{\mu}(x)-i \frac{\partial \mathcal{L}_{\text {total }}(x)}{\partial\left(\partial_{\mu} \phi_{I}\right)}\left(S_{\rho \sigma} \phi\right)_{I}=\frac{1}{2 m^{2}} H^{\mu \alpha \beta}\left(x_{\rho} \partial_{\sigma}-x_{\sigma} \partial_{\rho}\right) B_{\alpha \beta}-\left(F^{\mu \alpha}-B^{\mu \alpha}\right)\left(x_{\rho} \partial_{\sigma}-x_{\sigma} \partial_{\rho}\right) \\
& \times A_{\alpha}+C_{\alpha}\left(x_{\rho} \partial_{\sigma}-x_{\sigma} \partial_{\rho}\right) B^{\mu \alpha}-B\left(x_{\rho} \partial_{\sigma}-x_{\sigma} \partial_{\rho}\right) A^{\mu}-C\left(x_{\rho} \partial_{\sigma}-x_{\sigma} \partial_{\rho}\right) C^{\mu}-\left(x_{\rho} \delta_{\sigma}^{\mu}-x_{\sigma} \delta_{\rho}^{\mu}\right) \\
& \times\left[\frac{1}{12 m^{2}}\left(H_{\alpha \beta \gamma}\right)^{2}-\frac{1}{4}\left(F_{\alpha \beta}-B_{\alpha \beta}\right)^{2}+C^{\beta} \partial^{\alpha} B_{\alpha \beta}\right]+\left(\frac{1}{m^{2}} H^{\mu \alpha \beta}+\eta^{\mu \alpha} C^{\beta}-\eta^{\mu \beta} C^{\alpha}\right) \\
& \times\left(\eta_{\rho \alpha} B_{\sigma \beta}-\eta_{\sigma \alpha} B_{\rho \beta}\right)-\left(F^{\mu \alpha}-B^{\mu \alpha}+\eta^{\mu \alpha} B\right)\left(\eta_{\rho \alpha} A_{\sigma}-\eta_{\sigma \alpha} A_{\rho}\right)-\eta^{\mu \alpha} C\left(\eta_{\rho \alpha} C_{\sigma}-\eta_{\sigma \alpha} C_{\rho}\right),
\end{aligned}
$$

where the canonical energy-momentum tensor $T_{\rho}{ }^{\mu}(x)$ is given by

$$
\begin{aligned}
T_{\rho}{ }^{\mu}(x)= & \frac{1}{2 m^{2}} H^{\mu \alpha \beta} \partial_{\rho} B_{\alpha \beta}-\left(F^{\mu \alpha}-B^{\mu \alpha}\right) \partial_{\rho} A_{\alpha}+C_{\alpha} \partial_{\rho} B^{\mu \alpha} \\
& -B \partial_{\rho} A^{\mu}-C \partial_{\rho} C^{\mu}-\delta_{\rho}^{\mu}\left(\frac{1}{12 m^{2}}\left(H_{\alpha \beta \gamma}\right)^{2}-\frac{1}{4}\right. \\
& \left.\times\left(F_{\alpha \beta}-B_{\alpha \beta}\right)^{2}+C^{\beta} \partial^{\alpha} B_{\alpha \beta}\right) .
\end{aligned}
$$

From the conserved currents (3.2), one can obtain the generators of Lorentz transformation $M_{\rho \sigma}$ :

$$
M_{\rho \sigma}=\int d^{2} x M_{\rho \sigma}^{0}(x)
$$

By utilizing expressions (3.2) and (3.4) and the commutation relations (2.13), we have the nonvanishing VEV of the following commutation relations on the vacuum (3.1):

$$
\left\langle\left[i M_{0 i}, B_{0 j}(x)\right]\right\rangle=\int d^{2} \boldsymbol{y}\left\langle\left[i M_{0 i}^{0}(y), B_{0 j}(x)\right]\right\rangle=\epsilon_{i j} B_{\mathrm{VEV}},
$$

\footnotetext{
${ }^{10}$ Our convention for the Poincare algebra in $(2+1)$ dimensions is

$$
\begin{gathered}
{\left[P_{\mu}, P_{\nu}\right]=0, \quad\left[M_{\mu \nu}, P_{\rho}\right]=-i\left(\eta_{\mu \rho} P_{\nu}-\eta_{\nu \rho} P_{\mu}\right),} \\
{\left[M_{\mu \nu}, M_{\rho \sigma}\right]=-i\left(\eta_{\mu \rho} M_{\nu \sigma}-\eta_{\nu \rho} M_{\mu \sigma}+\eta_{\nu \sigma} M_{\mu \rho}-\eta_{\mu \sigma} M_{\nu \rho}\right) .}
\end{gathered}
$$
}

$$
\begin{aligned}
\left\langle\left[i M_{0 i}, F_{0 j}(x)\right]\right\rangle & =\int d^{2} \boldsymbol{y}\left\langle\left[i M_{0 i}{ }^{0}(y), F_{0 j}(x)\right]\right\rangle \\
& =\epsilon_{i j} B_{\mathrm{VEV}} \quad\left(\epsilon_{12}=1\right) .
\end{aligned}
$$

Thus two boost generators $M_{0 i}(i=1,2)$ are spontaneously broken on the vacuum. From the above commutation relations, the candidates for the NG bosons for the broken boost generators are $B_{0 i}$ and $F_{0 i}$. However, $B_{\mu \nu}$ is a mixture of massive and massless components as discussed previously. As obtained in the previous section, the mass eigenstates of the model are the massive field $U_{\mu \nu}$ and the massless field $F_{\mu \nu}\left(\right.$ or $A_{\mu}$ ) and the other massless fields $C_{\mu}, B$, and $C$. Since NG bosons are necessarily a massless state, we conclude that the NG bosons for the broken boost generators are the massless photon $F_{0 i}$. Incidentally, the massive field $U_{\mu \nu}$ satisfies

$$
\left\langle\left[i M_{0 i}, U_{\mu \nu}(x)\right]\right\rangle=\int d^{2} \boldsymbol{y}\left\langle\left[i M_{0 i}^{0}(y), U_{\mu \nu}(x)\right]\right\rangle=0 .
$$

Thus the fact that $B_{\mu \nu}$ becomes essentially a massive field is consistent with the Nambu-Goldstone theorem.

\section{B. Are the NG bosons physical?}

We have identified the NG bosons for the spontaneous Lorentz symmetry breaking with the massless photon $F_{0 i}$. The one-particle state of the massless photon $F_{\mu \nu}\left(\right.$ or $\left.A_{\mu}\right)$ is an unphysical state as discussed in Sec. III A. Hence the NG bosons for the spontaneous Lorentz symmetry breaking are unphysical states, i.e., unphysical NG bosons. Since the Lorentz symmetry is a physical global symmetry, the corresponding NG bosons are expected to be physical. Is this a contradiction?

\footnotetext{
${ }^{11}\left(S_{\rho \sigma} \phi\right)_{I}$ denotes infinitesimal transformations of internal spin: $\left(S_{\rho \sigma} \chi\right)=0$ for scalar fields, $\left(S_{\rho \sigma} V\right)_{\mu}=i\left(\eta_{\rho \mu} V_{\sigma}-\eta_{\sigma \mu} V_{\rho}\right)$ for vector fields, and $\left(S_{\rho \sigma} B\right)_{\mu \nu}=i\left(\eta_{\rho \mu} B_{\sigma \nu}-\eta_{\sigma \mu} B_{\rho \nu}+\eta_{\rho \nu} B_{\mu \sigma}\right.$ $-\eta_{\sigma \nu} B_{\mu \rho}$ ) for second-rank antisymmetric tensor fields.
} 
In order to answer this question, let us discuss the oneparticle state created by the broken boost generators from the vacuum (3.1). Inserting the decompositions $\phi_{I}(x)=\left\langle\phi_{I}\right\rangle$ $+\hat{\phi}_{I}(x)$ on the vacuum ${ }^{12}$ into the expressions of the currents (3.2), one can construct the broken parts of the currents $M_{0 i}^{B}{ }^{\mu}(x)$, which depends on $B_{\mathrm{VEV}}$, i.e., $M_{0 i}{ }^{\mu}$ $=M_{0 i}^{B}{ }^{\mu}\left(B_{\mathrm{VEV}}, \hat{\phi}_{I}\right)+\hat{M}_{0 i}{ }^{\mu}\left(\hat{\phi}_{I}\right)$. The explicit forms of the broken parts of the currents $M_{0 i}^{B}{ }^{\mu}(x)$ are

$$
\begin{aligned}
M_{0 i}^{B}{ }^{\mu}(x)= & B_{\mathrm{VEV}} \epsilon_{i j}\left\{\frac{1}{2} x^{j}\left(\hat{F}^{\mu 0}-\hat{B}^{\mu 0}+\eta^{\mu 0} \hat{B}\right)-\frac{1}{2} x_{0}\left(\hat{F}^{\mu j}\right.\right. \\
& \left.\left.-\hat{B}^{\mu j}+\eta^{\mu j} \hat{B}\right)+\left(\frac{1}{m^{2}} \hat{H}^{\mu 0 j}+\eta^{\mu 0} \hat{C}^{j}-\eta^{\mu j} \hat{C}^{0}\right)\right\}
\end{aligned}
$$

and $M_{12}^{B}{ }^{\mu}$ vanishes. Using the equations of motion for $\hat{\phi}_{I}$, which are the same as Eqs. (2.6)-(2.10), one can easily show that these currents are also conserved. From these currents one can obtain the broken parts of the generators, which are conserved:

$$
\begin{aligned}
M_{0 i}^{B} \equiv & \int d^{2} \boldsymbol{x} \quad M_{0 i}^{B}{ }^{0}(x) \\
= & B_{\text {vev }} \int d^{2} \boldsymbol{x} \quad \epsilon_{i j}\left\{\left[\frac{1}{2} x_{0} \hat{U}^{0 j}(x)\right]\right. \\
& \left.+\left[-\frac{1}{2} x_{0} \hat{C}^{0 j}(x)+\frac{1}{2} x^{j} \hat{B}(x)+\hat{C}^{j}(x)\right]\right\} .
\end{aligned}
$$

Hereafter we abbreviate simply $\hat{\phi}_{I}$ as $\phi_{I}$ without confusion. These broken parts of generators indeed satisfy

$$
\left[i M_{0 i}^{B}, B_{0 j}(x)\right]=\left[i M_{0 i}^{B}, F_{0 j}(x)\right]=\epsilon_{i j} B_{\mathrm{VEV}} .
$$

Note that $M_{0 i}^{B}$ is a physical operator that commutes with $C_{\mu}$, $B$, and $C$.

We consider the one-particle state created by $M_{0 i}^{B}$ from the vacuum (3.1), denoted as $|\mathrm{VAC}\rangle$. Since we are interested in the NG bosons for the broken generators, we consider only the massless state of the one-particle state given by

$$
\begin{aligned}
\left.M_{0 i}^{B}|\mathrm{VAC}\rangle\right|_{\text {massless }}= & B_{\mathrm{VEV}} \int d^{2} \boldsymbol{x} \epsilon_{i j}\left[-\frac{1}{2} x_{0} C^{0 j}(x)\right. \\
& \left.+\frac{1}{2} x^{j} B(x)+C^{j}(x)\right]|\mathrm{VAC}\rangle .
\end{aligned}
$$

From the commutation relations (2.13), one can easily find that these states are the physical states that satisfy the conditions (2.16), but the null states. Therefore we conclude that although the spontaneous Lorentz symmetry breaking is

\footnotetext{
${ }^{12}$ Since $\left\langle F_{12}\right\rangle=B_{\mathrm{VEV}}$, we take, for an example, the VEV's of $A_{i}$ as $\left\langle A_{1}\right\rangle=-\frac{1}{2} B_{\mathrm{VEV}} x^{2}$ and $\left\langle A_{2}\right\rangle=\frac{1}{2} B_{\mathrm{VEV}} x^{1}$.
}

physical, the massless one-particle state created by the broken generator $M_{0 i}^{B}$ from the vacuum (3.1), is not only a physical state but also a null state. Since our model has the twisted structure of the Hilbert space with an indefinite metric on account of the gauge invariance (2.3), nonzero matrix elements exist between physical null states and unphysical states. Thereby the NG boson, which has the nonzero matrix element with this massless one-particle state, can be unphysical. This is closely analogous to the Abelian Higgs model in the NL formalism [12].

As discussed in Sec. III A, in the limit $m \rightarrow 0$, the unphysical transverse photon, which is the unphysical NG boson for the spontaneous Lorentz symmetry breaking, becomes essentially the physical propagating state of the model. The implication of this fact will be discussed in Sec. IV.

\section{A PERTURBATION}

So far, we have studied the free field theory of $B_{\mu \nu}$ and $A_{\mu}$ whose action is given by Eq. (2.5). In this section, we discuss a possible perturbation of the model. We introduce the following interaction terms to Eq. (2.5) as the perturbation:

$$
\mathcal{L}_{\text {int }}(x)=\sum_{n=2}^{N} a_{n}\left\{\left(F_{\mu \nu}-B_{\mu \nu}\right)^{2}\right\}^{n}
$$

This type of interactions is obtained by the $\alpha^{\prime}$ expansion of DBI action. ${ }^{13}$ These interactions are gauge invariant under the gauge transformation (2.3) and consistent with the physical state conditions (2.16); these interaction terms do not change the equations of motion of $C_{\mu}, B$, and $C$ in Eq. (2.11) and keep them free fields. Although these interactions are nonrenormalizable, we treat them as the perturbations, which are interpreted as the operator insertions in the matrix elements in a similar manner to the chiral Lagrangian of QCD.

Although the equations of motion of $B_{\mu \nu}$ and $A_{\mu}$ is modified due to the interaction terms, the solution (3.1) still remains to be a solution of the modified equations. Thus, even after including the perturbations, the spontaneous Lorentz symmetry breaking is realized. In this case, the argument about the NG boson in the previous section does not essentially change and hence we can conclude that the NG boson remains to be the unphysical photon.

As is well-known, the low-energy dynamics of the NG boson is given by only the symmetry argument, i.e., the lowenergy theorem. In particular, the low-energy effective action of the NG boson is given by the nonlinear realization of the broken symmetry. In the case of the spontaneous Lorentz symmetry breaking, these argument holds and the lowenergy effective action of the NG boson is expected to be given by the nonlinear realization of the broken Lorentz symmetry. This problem will be argued in Sec. V.

\footnotetext{
${ }^{13} \mathrm{By}$ virtue of the peculiarity of the $(2+1)$ dimension, a Lorentz scalar constructed from $F_{\mu \nu}-B_{\mu \nu}$ can always be expressed as a polynomial of $\left(F_{\mu \nu}-B_{\mu \nu}\right)^{2}$.
} 
In the limit $m \rightarrow 0$, the physical propagating state becomes essentially the unphysical transverse photon. Furthermore the amplitude of the physical propagating state (2.26), for example, the two-point amplitude satisfies

$$
\begin{aligned}
& \left\langle\left[u_{01}(p)-u_{12}(p)\right] \widetilde{\mathcal{L}}_{\mathrm{int}}(q)\left[u_{01}^{+}(k)-u_{12}^{+}(k)\right]\right\rangle \\
& \quad=\left\langle\left[f_{01}(p)-f_{12}(p)\right] \widetilde{\mathcal{L}}_{\mathrm{int}}^{\left(b_{\mu \nu}=0\right)}(q)\left[f_{01}^{+}(k)-f_{12}^{+}(k)\right]\right\rangle,
\end{aligned}
$$

where $\widetilde{\mathcal{L}}_{\text {int }}(q)$ and $\widetilde{\mathcal{L}}_{\text {int }}^{\left(b_{\mu \nu}=0\right)}(q)$ are the Fourier transforms of $\mathcal{L}_{\text {int }}(x)$ and $\mathcal{L}_{\text {int }}^{\left(B_{\mu \nu}=0\right)}(x)$ which is obtained by setting $B_{\mu \nu}$ $=0$ in Eq. (4.1), respectively. This relation can be generalized to the scattering amplitudes for any number of the incoming or outgoing physical particles. Thus the physical amplitude in this limit is given by the amplitude of the unphysical transverse photon, that is, the NG boson of the spontaneous Lorentz symmetry breaking. This is an analog of the equivalence theorem of the Yang-Mills-Higgs model. In the energy region $\left(B_{\mathrm{VEV}}\right)^{2 / 3} \gg E \gg m$, where $\left(B_{\mathrm{VEV}}\right)^{2 / 3}$ is the scale of the Lorentz symmetry breaking, the physical $S$-matrix elements can be obtained by the scattering amplitudes of the NG bosons for the Lorentz symmetry breaking.

\section{DISCUSSIONS}

In this paper, we have studied a covariant canonical quantization of a gauge-invariant model of a second-rank antisymmetric tensor field and a vector field (photon). The spontaneous Lorentz symmetry breaking on the vacuum with a constant VEV of the antisymmetric tensor field has also been studied and the NG boson of the Lorentz symmetry breaking has been identified with the unphysical photon. In this section, we discuss some related problems.

\section{A. The spontaneous symmetry breaking of translation}

Until now, we have discussed only the spontaneous Lorentz symmetry breaking on the vacuum (3.1). Indeed, the vacuum breaks the translational symmetry, because the VEV $\left\langle F_{12}\right\rangle=B_{\mathrm{VEV}}$ leads to, for example, the VEV's

$$
\left\langle A_{1}\right\rangle=-\frac{1}{2} B_{\mathrm{VEV}} x^{2} \quad \text { and } \quad\left\langle A_{2}\right\rangle=\frac{1}{2} B_{\mathrm{VEV}} x^{1} .
$$

Since $A_{1}$ and $A_{2}$ are not gauge invariant, one may expect that the VEV's can be eliminated by a gauge transformation. However, following the discussion about the VEV's of $B_{\mu \nu}$ and $F_{\mu \nu}$ in Sec. III, we do not require the invariance of the Hilbert space under the singular gauge transformation which eliminates them.

By the VEV's (5.1), two translation generators $P_{1}$ and $P_{2}$ are broken:

$$
\left\langle\left[i P_{i}, A_{j}(x)\right]\right\rangle=\epsilon_{i j} \frac{1}{2} B_{\mathrm{VEV}},
$$

where $P_{i}$ is given by the canonical energy-momentum tensor (3.3):

$$
P_{i}=\int d^{2} x T_{i}^{0}(x)
$$

The NG bosons associated with the broken translation generator $P_{1}$ and $P_{2}$ are $A_{2}$ and $A_{1}$, respectively. A similar discussion with the broken Lorentz symmetry concludes that the NG boson of the broken translational symmetry is also the unphysical massless photon.

This can be also understood from the following commutation relation in the Poincaré algebra ${ }^{10}$

$$
\left[M_{0 i}, P_{0}\right]=-i P_{i} .
$$

Sandwiching the Jacobi identity

$$
\begin{aligned}
& {\left[\left[M_{0 i}, P_{0}\right], A_{j}(x)\right]+\left[\left[A_{j}(x), M_{0 i}\right], P_{0}\right]+\left[\left[P_{0}, A_{j}(x)\right], M_{0 i}\right]} \\
& \quad=0
\end{aligned}
$$

between the vacuum states $|\mathrm{VAC}\rangle$, one can obtain the following equality

$$
\left\langle\left[i P_{i}, A_{j}(x)\right]\right\rangle=\left\langle\left[i M_{0 i}, \partial_{0} A_{j}(x)\right]\right\rangle=\epsilon_{i j} \frac{1}{2} B_{\mathrm{VEV}},
$$

where we have used $P_{0}|\mathrm{VAC}\rangle=0$ and $\left[P_{0}, \phi_{I}\right]=-i \partial_{0} \phi_{I}$. This implies that when the NG boson of the broken translation generator $P_{i}$ is $A_{j}$, the NG boson of the broken Lorentz generator $M_{0 i}$ is given by its time derivative $\partial_{0} A_{j} \sim F_{0 j}$. This phenomenon has been known as the inverse Higgs phenomenon in the nonlinear realization of spacetime symmetries [20].

\section{B. Relation to the nonlinear realization of Lorentz symmetry}

According to the discussion in Sec. V A, the low-energy effective action of the NG boson $A_{i}$ can be obtained by the nonlinear realization of the translational and Lorentz symmetry, which leads to a one-dimensional effective action. ${ }^{14}$ However, since no physical lower-dimensional object such as a brane exists in our model, we expect a $(2+1)$ dimensional effective action, which describes the low-energy effective theory in the whole spacetime.

To realize this expectation, as in the case of the broken boost generators, we split the broken translation generators $P_{i}$ into the broken parts and the unbroken parts such as ${ }^{15}$ $P_{i}=P_{i}^{B}\left(B_{\mathrm{VEV}}\right)+\hat{P}_{i}$

$$
P_{i}^{B}=\frac{B_{\mathrm{VEV}}}{2} \int d^{2} \boldsymbol{x} \epsilon_{i j}\left(\hat{U}^{0 j}-\hat{C}^{0 j}\right) .
$$

The broken parts of the translation generators satisfy

\footnotetext{
${ }^{14}$ The Nambu-Goto type effective action on a lower-dimensional brane embedded in higher-dimensional flat spacetime is known to be obtained by the nonlinear realization of the higher-dimensional translational and Lorentz symmetries [21].

${ }^{15} \mathrm{As}$ in the case of the boost generators, one can show that $P_{i}^{B}$ and $\hat{P}_{i}$ are conserved separately.
} 


$$
\left[i P_{i}^{B}, A_{j}\right]=\epsilon_{i j} \frac{1}{2} B_{\mathrm{VEV}} .
$$

The same argument as the case of the boost generators leads us to the conclusion that the NG bosons for the broken translation generators can be the unphysical massless photon.

Here, if one considers only the physical Hilbert space defined by the physical state conditions (2.16), one can show that the generators $\left\{M_{\mu \nu}, P_{0}, \hat{P}_{i}\right\}$ form a closed Poincaré algebra on the physical Hilbert space. As far as one considers this Poincare algebra on the physical Hilbert space, the translational symmetry generated by $\hat{P}_{i}$ is unbroken and only the boost symmetry generated by $M_{0 i}$ is broken. In this breaking pattern of the Poincare symmetry, the low-energy effective action constructed by the nonlinear realization is a $(2+1)$ dimensional effective action and its explicit form has been obtained in Ref. [19]. Thus, we expect that the physical amplitudes of the model can be obtained by the low-energy effective action via the equivalence theorem discussed in Sec. V A.

\section{Other related topics}

From the relation between the noncommutative gauge theory and the D-brane world volume effective theory on a constant background antisymmetric tensor field, our investigation is expected to give some new insights to the noncommutative gauge theory. However, in this context, the antisymmetric tensor field is taken as an external field; the kinetic term for the antisymmetric tensor field does not exist. Then the vacuum

$$
\left\langle B_{12}\right\rangle \neq 0 \quad \text { and } \quad\left\langle F_{12}\right\rangle=0
$$

[1] A. Connes, M. R. Douglas, and A. Schwarz, J. High Energy Phys. 02, 3 (1998).

[2] M. R. Douglas and C. Hull, J. High Energy Phys. 02, 8 (1998).

[3] H. Aoki, N. Ishibashi, S. Iso, H. Kawai, Y. Kitazawa, and T. Tada, Nucl. Phys. B565, 176 (2000).

[4] N. Seiberg and E. Witten, J. High Energy Phys. 09, 32 (1999).

[5] M. Gasperini, Phys. Rev. D 33, 3594 (1986).

[6] M. Kalb and P. Ramond, Phys. Rev. D 9, 2273 (1974).

[7] E. Cremmer and J. Scherk, Nucl. Phys. B72, 117 (1974).

[8] C. G. Callan, C. Lovelace, C. R. Nappi, and S. A. Yost, Nucl. Phys. B288, 525 (1987).

[9] M. Green, Phys. Lett. B 302, 29 (1993).

[10] For example, J. Polchinski, String Theory (Cambridge University, Cambridge, England, 1998), Vols. I and II.

[11] N. Nakanishi, Suppl. Prog. Theor. Phys. 51, 1 (1972).

[12] N. Nakanishi and I. Ojima, Covariant Operator Formalism of Gauge (World Scientific, Singapore, 1990) and references is allowed as the solution of the equation of motion of a gauge field $A_{\mu}$. This solution is a different vacuum from our vacuum (3.1). The extension of our analysis to the above case and the relation to the noncommutative gauge theory are interesting problems.

We make some speculations about the dynamics of photon $A_{\mu}$ in our model. At first, since the photon is the NG boson of the broken Lorentz symmetry on the vacuum (3.1), the NG theorem concludes that the photon cannot become massive on the vacuum. Second, as discussed in Ref. [19], the NG boson of the broken Lorentz symmetry has derivative couplings with any fields including itself. Hence, the photon is expected to have derivative coupling with any fields including itself and neutral fields. From the relation to the noncommutative gauge theory, this seems consistent with the fact that the noncommutative photon in the noncommutative QED, where the products of fields are replaced by the $\star$ products, has self-couplings and derivative couplings with fields in the adjoint representation, i.e., neutral fields [22,23].

Although the discussion in this paper is limited to the model in $2+1$ dimensions, similar discussions can be applied to the models in higher dimensions. Investigation of the more realistic case, that is, the $(3+1)$-dimensional case is an interesting future problem.

Investigation of the supersymmetric extension of our model from the viewpoint in this paper is also interesting.

\section{ACKNOWLEDGMENTS}

The authors would like to thank N. Ohta, T. Nakatsu, M. Torii, T. Kimura, and T. Kugo for useful discussions and comments.

therein.

[13] N. Nakanishi, Prog. Theor. Phys. 51, 952 (1974).

[14] E. C. G. Stueckelberg, Helv. Phys. Acta 11, 299 (1938).

[15] M. S. Chanowitz and M. K. Gaillard, Nucl. Phys. B261, 379 (1985).

[16] K. Aoki, On the Equivalence Theorem Between $W(L), Z(L)$ and Nambu-Goldstone Particles, talk presented at the meeting on Physics at TeV Energy Scale (KEK, Tsukuba, 1987).

[17] V. A. Kostelecký and S. Samuel, Phys. Rev. D 39, 683 (1989).

[18] V. A. Kostelecký and S. Samuel, Phys. Rev. D 42, 1289 (1990).

[19] N. Yokoi, Phys. Lett. B 504, 109 (2001).

[20] E. A. Ivanov and V. I. Ogievetsky, Teor. Mat. Fiz. 25, 164 (1975).

[21] E. Ivanov, hep-th/0002204.

[22] M. Hayakawa, Phys. Lett. B 478, 394 (2000).

[23] M. Hayakawa, hep-th/9912167. 